PAR LPTHE 92/32; hep - th/9209047

\title{
EXACT STRING SOLUTIONS IN 2+1-DIMENSIONAL DE SITTER SPACETIME
}

\author{
H.J. DE VEGA \\ Laboratoire de Physique Théorique et Hautes Energies ${ }^{\star}$, Paris
}

\begin{abstract}
A. V. Mikhailov
Landau Institute for Theoretical Physics, Russian Academy of Sciences ${ }^{\dagger}$ and Observatoire de Paris, Section de Meudon, Demirm*
\end{abstract}

\author{
N. SÁNCHEZ \\ Observatoire de Paris, Section de Meudon, Demirm ${ }^{\ddagger}$
}

* Laboratoire Associé au CNRS UA 280

Postal address:

L.P.T.H.E., Tour 16, $1^{\mathrm{er}}$ étage, Université Paris VI, 4. Place Jussieu, 75252, Paris cedex 05, FRANCE.

$\dagger$ Ul. Kossyguina 2, 117334 Moscow, RUSSIA

¥Laboratoire Associé au CNRS UA 336, Observatoire de Meudon et École Normale Supérieure.

Postal address:

DEMIRM, Observatoire de Paris. Section de Meudon, 92195 MEUDON Principal Cedex, FRANCE. 


\section{ABSTRACT}

Exact and explicit string solutions in de Sitter spacetime are found. (Here, the string equations reduce to a sinh-Gordon model). A new feature without flat spacetime analogy appears: starting with a single world-sheet, several (here two) strings emerge. One string is stable and the other (unstable) grows as the universe

grows. Their invariant size and energy either grow as the expansion factor or tend to constant. Moreover, strings can expand (contract) for large (small) universe radius with a different rate than the universe. 
String propagation in curved spacetimes reveals new insights for string theory. The string behaviour in strong gravitational fields and in the vicinity of spacetime singularities is especially interesting. In gravitational shock-waves and singular plane wave spacetimes the string equations are exactly (and explicitly) solvable even at the spacetime singularities uncovering a rich variety of physical phenomena[1]. The classical string propagation in D-dimensional de Sitter spacetime is an integrable model as it has been explicitly shown in ref.[3] where, in addition, all the $1+1$ dimensional string solutions were given. It is clearly an appealing challenge to explicitly solve the string propagation in such a relevant cosmological spacetime as the de Sitter universe.

The string solutions reported here indeed apply to cosmic strings in de Sitter spacetime as well. The dynamics of cosmic strings in expanding universes has been studied in the literature for the Friedman-Robertson-Walker (FRW) cases (see for example $[2,7,8]$ ). It must be noticed that the string behaviour we found here in de Sitter universe is essentially different from the standard FRW where $R\left(t_{0}\right)$ is a positive power of the cosmic time $t_{0}$. In such FRW universes, strings always oscillate in time, the comoving spatial string coordinates contract and the proper string size stays constant asymptotically for $t_{0} \rightarrow \infty$. In the cosmic string literature this is known as 'string stretching'. We called such behaviour 'stable' [6]. On the contrary, in de Sitter spacetime, as we show below, two types of asymptotic behaviors are present : (i) the proper string size and energy grow with the expansion factor ('unstable' behaviour) or (ii) they tend to constant values ('stable' strings).

The unstable string solutions in de Sitter universe may provide a mechanism to self-sustain inflation as proposed in refs.[9]-[6] without advocating an inflaton field. The present note is a first step in the investigation of multi-string exact solutions in de Sitter spacetime using soliton methods [4]. Such general solutions should provide essential clues about the feasability of inflationary string scenarios.

In this letter we present exact and explicit solutions for strings propagating 
in the $2+1$ dimensional de Sitter spacetime. In this case, de Sitter spacetime can be considered as a 3-dimensional hyperboloid embedded in a 4 dimensional flat Minkowski spacetime with coordinates $\left(q_{0}, q_{1}, q_{2}, q_{3}\right)$ and metric

$$
d s^{2}=\frac{1}{H^{2}}\left[-\left(d q^{0}\right)^{2}+\left(d q^{1}\right)^{2}+\left(d q^{2}\right)^{2}+\left(d q^{3}\right)^{2}\right]
$$

where $\mathrm{H}$ is Hubble's constant and

$$
\left(q^{0}\right)^{2}=\left(q^{1}\right)^{2}+\left(q^{2}\right)^{2}+\left(q^{3}\right)^{2}-1
$$

In the comoving coordinates $\left(t_{0}, X^{1}, X^{2}\right)$ the de Sitter metric takes the form

$$
d s^{2}=-\left(d t_{0}\right)^{2}+e^{2 H t_{0}}\left[\left(d X^{1}\right)^{2}+\left(d X^{2}\right)^{2}\right]
$$

where the cosmic time $t_{0}$ and the conformal time $\eta$ are given by

$$
\eta=-\frac{1}{H} \exp \left[-H t_{0}\right]=-\frac{1}{H\left(q_{0}+q_{1}\right)}
$$

and

$$
X^{1}=\frac{q^{2}}{H\left(q^{0}+q^{1}\right)} \quad, \quad X^{2}=\frac{q^{3}}{H\left(q^{0}+q^{1}\right)},
$$

The string equations of motion take here the form

$$
\frac{\partial^{2} q}{\partial x_{-} \partial x_{+}}+\left[\frac{\partial q}{\partial x_{+}} \cdot \frac{\partial q}{\partial x_{-}}\right] q=0
$$

where . stands for the Lorentzian scalar product $a . b \equiv-a_{0} b_{0}+a_{1} b_{1}+a_{2} b_{2}+a_{3} b_{3}$ $, x_{ \pm} \equiv \frac{1}{2}(\tau \pm \sigma), \sigma$ and $\tau$ being the string world sheet coordinates. In addition, 
we have eq.(2), i.e. $q \cdot q=1$, and the string constraints on the world sheet are

$$
T_{ \pm \pm}=\frac{\partial q}{\partial x_{ \pm}} \cdot \frac{\partial q}{\partial x_{ \pm}}=0
$$

We define

$$
\exp [\alpha(\sigma, \tau)]=-\frac{\partial q}{\partial x_{-}} \cdot \frac{\partial q}{\partial x_{+}}
$$

As it is shown in ref.[3], $\alpha(\sigma, \tau)$ obeys the sh-Gordon equation

$$
\frac{\partial^{2} \alpha}{\partial \tau^{2}}-\frac{\partial^{2} \alpha}{\partial \sigma^{2}}-\exp \alpha+\exp -\alpha=0
$$

Notice that for closed strings $q(\sigma, \tau)$ and hence $\alpha(\sigma, \tau)$ are periodic functions of $\sigma$ with period $2 \pi$. Therefore, to find string solutions in de Sitter spacetime we can start from a periodic solution of eq.(9), and insert it on the field equations (6):

$$
\left[\frac{\partial^{2}}{\partial \tau^{2}}-\frac{\partial^{2}}{\partial \sigma^{2}}-\exp \alpha(\sigma, \tau)\right] q(\sigma, \tau)=0
$$

Once this linear equation in $q(\sigma, \tau)$ is solved, it remains to impose the constraints (7) and eq.(8).

Let us remark that $\exp [\alpha(\sigma, \tau)]$ has a clear physical interpretation. The invariant interval between two points on the string computed with the spacetime metric (1) is given by

$$
d s^{2}=\frac{1}{H^{2}} d q \cdot d q=\frac{1}{2 H^{2}} \exp [\alpha(\sigma, \tau)]\left(d \sigma^{2}-d \tau^{2}\right)
$$

Therefore we can define

$$
S(\sigma, \tau) \equiv \frac{1}{\sqrt{2} H} \exp [\alpha(\sigma, \tau) / 2]
$$

as the invariant string size. The energy density for the sinh-Gordon model reads 
here

$$
\mathcal{H}=\frac{1}{2}\left[\left(\frac{\partial \alpha}{\partial \tau}\right)^{2}+\left(\frac{\partial \alpha}{\partial \sigma}\right)^{2}\right]-2 \cosh \alpha
$$

That means a potential unbounded from below

$$
V_{e f f}=-2 \cosh \alpha
$$

with absolute minima at $\alpha=+\infty$ and at $\alpha=-\infty$. As the time $\tau$ evolves, $\alpha(\sigma, \tau)$ will generically approach these infinite minima. The first minimum corresponds to an infinitely large string whereas the second describes a collapsed situation. That means that strings in de Sitter spacetime will generically tend either to inflate at the same rate as the universe (when $\alpha \rightarrow+\infty$ ) or to collapse to a point (when $\alpha \rightarrow-\infty)$. As we shall see below these general trends are confirmed by the explicit string solutions. Let us start by studying solutions where $\alpha=\alpha(\tau)$. Then, the energy is

$$
\frac{1}{2} \alpha^{\prime 2}-2 \cosh \alpha=E=\text { constant }
$$

$\alpha(\tau)$ describes the position of a non-relativistic particle with unit mass rolling down the effective potential $V_{\text {eff }}=-2 \cosh \alpha$. A particularly interesting situation is the critical case $E=-2$ when one starts to roll down from the maximun of $V_{\text {eff }}$. That is, the initial speed is zero and the 'time' $\tau$ to reach either minimun $(\alpha=\infty$ or $-\infty)$ is infinity. The corresponding solutions are

$$
\alpha_{-}(\tau)=\log \left[\operatorname{coth}^{2}\left(\frac{\tau}{\sqrt{2}}\right)\right] \quad \text { and } \quad \alpha_{+}(\tau)=\log \left[\tanh ^{2}\left(\frac{\tau}{\sqrt{2}}\right)\right]
$$

$\alpha_{-}(\tau)$ starts at $\alpha=0$ for $\tau=-\infty$ and rolls down to the right reaching $\alpha=+\infty$ for $\tau \rightarrow 0^{-}$. The behaviour of $\alpha_{-}(\tau)$ near the initial and final points is as follows:

$$
\begin{gathered}
\alpha_{-}(\tau) \stackrel{\tau \rightarrow-\infty}{=} 4 e^{\tau \sqrt{2}}+O\left(e^{2 \tau \sqrt{2}}\right) \\
\alpha_{-}(\tau) \stackrel{\tau \rightarrow 0}{=} \log \frac{2}{\tau^{2}}+\frac{1}{3} \tau^{2}+O\left(\tau^{4}\right)
\end{gathered}
$$

The solution $\alpha_{+}(\tau)$ also starts at $\alpha=0$ for $\tau=-\infty$ but rolls down to the left 
reaching $\alpha=-\infty$ for $\tau \rightarrow 0^{-}$. We have for $\alpha_{+}(\tau)$ :

$$
\begin{array}{ll}
\alpha_{+}(\tau) & \stackrel{\tau \rightarrow-\infty}{=}-4 e^{\tau \sqrt{2}}+O\left(e^{2 \tau \sqrt{2}}\right) \\
\alpha_{+}(\tau) & \stackrel{\tau \rightarrow 0}{=}-\log \frac{2}{\tau^{2}}-\frac{1}{3} \tau^{2}+O\left(\tau^{4}\right)
\end{array}
$$

Notice that $\alpha_{+}(\tau)=-\alpha_{-}(\tau)$. In addition we have the trivial (but exact) solution $\alpha^{(o)}(\tau) \equiv 0$. Now that the function $\alpha(\tau)$ is known, we proceed to solve eq.(10) for $q(\sigma, \tau)$ with the constraints $(7)$ and (8). Since $q_{0}$ is a time-like coordinate, we shall assume $q_{0}=q_{0}(\tau)$. A natural ansatz is then

$$
q=\left(q_{0}(\tau), q_{1}(\tau), f(\tau) \cos \sigma, f(\tau) \sin \sigma\right)
$$

Then, eqs.(2) and (6) - (8) require

$$
\begin{gathered}
q_{0}(\tau)^{2}=q_{1}(\tau)^{2}+f(\tau)^{2} \\
{\left[\frac{d q_{0}}{d \tau}\right]^{2}=\left[\frac{d q_{1}}{d \tau}\right]^{2}+\left[\frac{d f}{d \tau}\right]^{2}+f^{2}} \\
e^{\alpha(\tau)}=\left[\frac{d q_{0}}{d \tau}\right]^{2}-\left[\frac{d q_{1}}{d \tau}\right]^{2}-\left[\frac{d f}{d \tau}\right]^{2}+f^{2}
\end{gathered}
$$

and

$$
\begin{aligned}
\frac{d^{2}}{d^{2} \tau} q_{0}-e^{\alpha(\tau)} q_{0}(\tau) & =0 \\
\frac{d^{2}}{d^{2} \tau} q_{1}-e^{\alpha(\tau)} q_{1}(\tau) & =0 \\
\frac{d^{2}}{d^{2} \tau} f(\tau)+f(\tau)-e^{\alpha(\tau)} f(\tau) & =0
\end{aligned}
$$

In addition, it seems reasonable to choose the time coordinate $q_{0}(\tau)$ to be an odd function of $\tau$. Remarkably enough, eqs.(20) - (23) admit consistent solutions for 
$\alpha(\tau)=\alpha_{+}(\tau), \alpha(\tau)=\alpha_{-}(\tau)$ and $\alpha(\tau)=0$. For $\alpha(\tau)=\alpha^{(o)}(\tau)=0$, we find

$$
q^{(o)}(\sigma, \tau)=\frac{1}{\sqrt{2}}(\sinh \tau, \cosh \tau, \cos \sigma, \sin \sigma)
$$

For $\alpha(\tau)=\alpha_{-}(\tau)$, we have

$$
\begin{gathered}
q_{-}(\sigma, \tau)=\left(\sinh \tau-\frac{1}{\sqrt{2}} \cosh \tau \operatorname{coth}\left[\frac{1}{\sqrt{2}} \tau\right], \quad \cosh \tau-\frac{1}{\sqrt{2}} \sinh \tau \operatorname{coth}\left[\frac{1}{\sqrt{2}} \tau\right]\right. \\
\left.\frac{1}{\sqrt{2}} \cos \sigma \operatorname{coth}\left[\frac{1}{\sqrt{2}} \tau\right], \quad \frac{1}{\sqrt{2}} \sin \sigma \operatorname{coth}\left[\frac{1}{\sqrt{2}} \tau\right]\right)
\end{gathered}
$$

And for $\alpha(\tau)=\alpha_{+}(\tau)$ we find

$$
\begin{gathered}
q_{+}(\sigma, \tau)=\left(\sinh \tau-\frac{1}{\sqrt{2}} \cosh \tau \tanh \left[\frac{1}{\sqrt{2}} \tau\right], \quad \cosh \tau-\frac{1}{\sqrt{2}} \sinh \tau \tanh \left[\frac{1}{\sqrt{2}} \tau\right]\right. \\
\left.\frac{1}{\sqrt{2}} \cos \sigma \tanh \left[\frac{1}{\sqrt{2}} \tau\right], \quad \frac{1}{\sqrt{2}} \sin \sigma \tanh \left[\frac{1}{\sqrt{2}} \tau\right]\right)
\end{gathered}
$$

These string solutions are given for a fixed de Sitter frame. Applying the de Sitter group to them yields a multi-parameter family of solutions. As it is clear, we can study them in the frame corresponding to eqs.(24) - (26) without loss of generality. Let us now discuss the physical interpretation of these solutions.

The string energy can be easily computed from the spacetime string energymomentum tensor:

$$
\sqrt{-G} T^{A B}(X)=\frac{1}{2 \pi \alpha^{\prime}} \int d \sigma d \tau\left(\dot{X}^{A} \dot{X}^{B}-X^{A} X^{\prime B}\right) \delta^{(D)}(X-X(\sigma, \tau))
$$

Whenever $t_{0}=t_{0}(\tau)$, the string energy at a time $t_{0}$ is given by:

$$
E\left(t_{0}\right)=\int d^{D-1} X \sqrt{-G} T^{00}(X)=\frac{1}{\alpha^{\prime}} \frac{d t_{0}}{d \tau}
$$

where $\alpha^{\prime}$ stands for the string tension. 
We recall that for a given time $q_{0}=q_{0}(\tau)$, the de Sitter space is a sphere $S^{2}$ with radius $R(\tau)=\frac{1}{H} \sqrt{1+q_{0}(\tau)^{2}}$. The solution $q^{(o)}(\sigma, \tau)$ [eq.(24)], describes a string of constant size $S^{(o)}=\frac{1}{\sqrt{2} H}$ and constant energy $E^{(o)}=\frac{1}{\alpha^{\prime} H}$, in a de Sitter universe that inflates for $\tau \rightarrow \infty$ since for this solution $R(\tau)=\frac{1}{H} \sqrt{1+\frac{\sinh ^{2} \tau}{2}}$. This solution is probably unstable under small perturbations.

Let us analyze now the solution $q_{-}(\sigma, \tau)$. Fig. 1 depicts the time coordinate $q_{-}(\sigma, \tau)_{0}$. We see that this solution describes actually two strings, since for a given value of $q_{0}$, there are two values of $\tau$. That is, $\tau$ is a two-valued function of $q_{0}$. Each branch of $\tau$ as a function of $q_{0}$ (or $t_{0}$ ) corresponds to a different string. This an entirely new feature for strings in curved spacetime. It has no analogy in flat spacetime where the time coordinate obeys the D'Alambert equation and therefore one can always choose a gauge where the time is proportional to $\tau$. The appearence of multiple strings is a generic feature in de Sitter spacetime as shown in ref.[4], where exact multistring solutions are constructed. For this solution $q_{-}(\sigma, \tau)$, the string size and energy are

$$
S_{-}(\tau)=\frac{1}{\sqrt{2} H} \operatorname{coth}\left|\frac{1}{\sqrt{2}} \tau\right|, \quad E_{-}(\tau)=\frac{1}{\alpha^{\prime} H}\left|1+\frac{1}{\cosh (\sqrt{2} \tau)-\frac{1}{\sqrt{2}} \sinh (\sqrt{2} \tau)-1}\right|
$$

That is, the string size increases for $\tau<0$ and decreases for $\tau>0$ with a singular behaviour $\frac{1}{|\tau|}$ for $\tau \rightarrow 0$.

We first analyze the inflationary expansion phase $q_{0}>0$. For $q_{0}=0$, the two strings correspond to (we call them I and II) :

$$
\begin{aligned}
\operatorname{String}(I): & \tau=-\tau_{0} \\
\operatorname{String}(I I): & \tau=+\tau_{0}
\end{aligned}
$$

where $\tau_{0}=1.4890 \ldots$ is the positive root of $q_{-0}(\tau)=0$ (see eq. $(25)$ ). Both strings have at $q_{0}=0$ the same size

$$
S_{-}\left( \pm \tau_{0}\right)=\frac{.903 .}{H}
$$

and different energies, $E_{-}\left(\tau_{0}\right)=\frac{4.260 \ldots}{\alpha^{\prime} H}>E_{-}\left(-\tau_{0}\right)=\frac{1.166 \ldots}{\alpha^{\prime} H}$. 
For $q_{0} \rightarrow+\infty$, the two strings correspond to

$$
\begin{aligned}
\operatorname{String}(I): & \tau=-\frac{1}{q_{0}} \rightarrow 0^{-} \\
\operatorname{String}(I I): & \tau=\log q_{0}-\log \left[\frac{1}{2}\left(1-\frac{1}{\sqrt{2}}\right)\right] \rightarrow+\infty
\end{aligned}
$$

Asymptotically, the string sizes and energies are

$$
\begin{array}{ll}
(I): \quad S_{-} & \stackrel{q_{0} \rightarrow \infty}{=} \frac{1}{H|\tau|} \simeq \frac{R\left(q_{0}\right)}{H} \rightarrow+\infty \\
& E_{-} \stackrel{q_{0} \rightarrow \infty}{=} \frac{1}{\alpha^{\prime} H}\left(\frac{1}{|\tau|}+1\right) \simeq \frac{1}{\alpha^{\prime} H}\left(R\left(q_{0}\right)+1\right) \rightarrow+\infty . \\
(I I): & S_{-} \stackrel{q_{0} \rightarrow \infty}{=} \frac{1}{\sqrt{2} H} \quad, \quad E_{-} \stackrel{q_{0} \rightarrow \infty}{=} \frac{1}{\alpha^{\prime} H}
\end{array}
$$

We see that for the string (I) both its size and energy grow monotonically, this growing becoming explosive for $q_{0} \rightarrow \infty$ when the size of the de Sitter space diverges. Actually, the string grows there at the same rate as the whole space. This describes an unstable string. The branch II represents a stable string for $q_{0} \rightarrow \infty$, both size and energy being asymptotically constant. It must be noticed that the size and the energy of the string I monotonically increase with $q_{0}$ whereas for the string II, $S_{-}$and $E_{-}$both monotonically decrease with $q_{0}$.

Let us describe now the solution $q_{-}(\sigma, \tau)$ for $q_{0}<0$, that is in the contracting phase of de Sitter universe. For $q_{0} \rightarrow-\infty$ we have for the strings I and II :

$$
\begin{aligned}
& (I): \quad \tau=-\log \left(-q_{0}\right)+\log \left[\frac{1}{2}\left(1-\frac{1}{\sqrt{2}}\right)\right] \rightarrow-\infty \\
& (I I): \quad \tau=-\frac{1}{q_{0}} \rightarrow 0^{+}
\end{aligned}
$$

The string size and energy are in this limit:

$$
\begin{aligned}
(I): & S_{-} \stackrel{q_{0} \rightarrow-\infty}{=} \frac{1}{\sqrt{2} H} \quad, \quad E_{-} \stackrel{q_{0} \rightarrow-\infty}{=} \frac{1}{\alpha^{\prime} H} \\
(I I): & S_{-} \stackrel{q_{0} \rightarrow-\infty}{=} \frac{1}{H \tau} \simeq \frac{R\left(q_{0}\right)}{H} \rightarrow+\infty \\
& E_{-} \stackrel{q_{0} \rightarrow-\infty}{=} \frac{1}{\alpha^{\prime} H}\left(\frac{1}{\tau}+1\right) \simeq \frac{1}{\alpha^{\prime} H}\left(R\left(q_{0}\right)+1\right) \rightarrow+\infty .
\end{aligned}
$$


The string I starts in the contracting phase with a stable behaviour for $q_{0} \rightarrow-\infty$, while string II starts with an infinite size (the size of the de Sitter space) for $q_{0} \rightarrow-\infty$. The size and energy of the string I grow monotonically with $q_{0}$. String II contracts with the universe itself for $q_{0}<0$ and continues to contract for $q_{0}>0$ until reaches the constant value $S_{-}=\frac{1}{\sqrt{2} H}$ for $q_{0} \rightarrow \infty$.

The behaviour for small $|\tau|$ confirms the asymptotic results found in refs. [3- 5$6]$.

It is interesting to study this string solution in the comoving de Sitter coordinates. The cosmic time $t_{0}$ and the conformal time $\eta$ [eq.(4)] take for $q_{-}(\sigma, \tau)$ the form:

$$
\begin{gathered}
e^{H t_{0}}=-\frac{1}{H \eta}=\left|1-\frac{1}{\sqrt{2}} \operatorname{coth}\left(\frac{1}{\sqrt{2}} \tau\right)\right| e^{\tau} \\
\rho=\frac{e^{-\tau}}{\left|1-\sqrt{2} \tanh \left(\frac{1}{\sqrt{2}} \tau\right)\right|}
\end{gathered}
$$

where

$$
\rho \equiv \sqrt{\left(X^{1}\right)^{2}+\left(X^{2}\right)^{2}}
$$

Therefore, for $t_{0} \rightarrow+\infty$, we have :

$$
\begin{aligned}
\operatorname{string}(I): \quad \eta & =\frac{\tau}{H} \rightarrow 0^{-}, \quad \rho=\frac{1}{H}+O\left(\tau^{2}\right) \\
t_{0} & =-\frac{1}{H} \log |\tau|+O(\tau) \rightarrow+\infty \\
\operatorname{string}(I I): \quad \eta & =-\frac{2+\sqrt{2}}{H} e^{-\tau} \rightarrow 0^{-}, \rho=\frac{e^{-\tau}}{H(\sqrt{2}-1)} \rightarrow 0 \\
t_{0} & =\frac{\tau}{H}+\frac{1}{H} \log \left(1-\frac{1}{\sqrt{2}}\right) \rightarrow+\infty
\end{aligned}
$$

We see that in the unstable regime (string I for $t_{0} \rightarrow+\infty$ ), the comoving string coordinates $\left(X^{1}, X^{2}\right)$ stay constant whereas the proper string size $S_{-}$and the energy $E_{-}$blow up [see eqs.(30)]. In this regime $\tau$ is proportional to the conformal time $\eta$. On the other hand, in the stable regime (string II for $t_{0} \rightarrow+\infty$ ), the 
comoving string coordinates $\left(X^{1}, X^{2}\right)$ vanish and $S_{-}$and $E_{-}$keep constant, [see eqs.(30)]. In this stable regime, $\tau$ is proportional to the cosmic time $t_{0}$. Notice that these results confirm the asymptotic behavior (34) discussed in previous works $[3$, $5,6]$.

Let us now discuss the solution $q_{+}(\sigma, \tau)$ [eq.(26)]. Here $\tau$ is a single value function of $q_{0}$, and hence this solution describes only one string.

There are two phases here:

(i) $q_{0}<0$ i. e. $\tau<0$ : contraction phase, $R(\tau)$ decreases,

(ii) $q_{0}>0$ i. e. $\tau>0$ : expansion phase, $R(\tau)$ grows.

Here,

$$
q_{0} \stackrel{\tau \rightarrow \pm \infty}{=} \pm \frac{1}{2}\left(1-\frac{1}{\sqrt{2}}\right) e^{ \pm \tau} \rightarrow \pm \infty
$$

The string size and energy are here

$$
\begin{aligned}
& S_{+}(\tau)=\frac{1}{\sqrt{2} H} \tanh \left|\frac{1}{\sqrt{2}} \tau\right| \\
& E_{+}(\tau)=\frac{1}{\alpha^{\prime} H}\left[1-\frac{1}{\cosh (\sqrt{2} \tau)+1-\frac{1}{\sqrt{2}} \sinh (\sqrt{2} \tau)}\right]
\end{aligned}
$$

Therefore, the string contracts from a fixed size $S_{+}=\frac{1}{\sqrt{2} H}$ and energy $E_{+}=\frac{1}{\alpha^{\prime} H}$ at $q_{0}=-\infty$ during (i) until the collapse at $q_{0}=0$ where $S_{+}$vanishes but not the energy which takes the value $E_{+}=\frac{1}{2 \alpha^{\prime} H}$. At this point the de Sitter space has a minimun size $\frac{1}{H}$. For $q_{0}>0$, the string size grows from zero until it takes the value $S_{+}=\frac{1}{\sqrt{2} H}$ for $q_{0} \rightarrow \infty$, and the energy reaches again the value $E_{+}=\frac{1}{\alpha^{\prime} H}$, while the de Sitter space radius tends to infinity as

$$
R_{+}\left(q_{0}\right) \stackrel{q_{0} \rightarrow \infty}{=} \frac{q_{0}}{H} \stackrel{q_{0} \rightarrow \infty}{=}\left(1-\frac{1}{\sqrt{2}}\right) \frac{1}{2 H} e^{\tau}
$$

This behaviour is different from $q_{-}(\sigma, \tau)$ and was not found before. Additional 
solutions follow by replacing

$$
\sigma \rightarrow n \sigma, \quad \tau \rightarrow n \tau, \quad n \in Z
$$

in eqs.(24) - (26) . In these solutions the string is winded $n$ times around the $q_{1}$ axis.

Strings propagating in de Sitter spacetime enjoy as conserved quantities those associated with the $\mathrm{O}(3,1)$ rotations on the hyperboloid (2) . They can be written as

$$
L_{A B}=\int_{0}^{2 \pi} d \sigma\left(q_{A} \dot{q}_{B}-q_{B} \dot{q}_{A}\right) \quad, \quad 0 \leq A, B \leq 3
$$

where $L_{A B}=-L_{B A}$. For the solutions $q^{(o)}, q_{-}$and $q_{+}$, only $L_{01}$ does not vanish, taking the value:

$$
L_{01}=-L_{10}=n \pi
$$

\section{REFERENCES}

1. H.J. de Vega and N. Sánchez, Lectures delivered at the Erice School "String Quantum Gravity and Physics at the Planck scale", 21-28 June 1992, Proceedings edited by N. Sánchez, World Scientific, 1993.

2. See for a review, T.W.B. Kibble, Erice Lectures at the Chalonge School in Astrofundamental Physics, N. Sánchez editor, World Scientific, 1992.

3. H. J. de Vega and N. Sánchez, Phys. Rev. D 47, 3394 (1993).

4. F. Combes, H.J. de Vega, A.V. Mikhailov and N. Sánchez, lpthe preprint $93 / 44$.

5. H. J. de Vega and N. Sánchez, Phys. Lett. 197B, 320 (1987). 
6. N. Sánchez and G. Veneziano, Nucl. Phys. B333, 253 (1990), M. Gasperini, N.Sánchez and G. Veneziano, Int. J. Mod. Phys. A6, 3853 (1991) and Nucl. Phys. B364, 365 (1991).

7. A. Vilenkin, Phys. Rev. D 24, 2082 (1981), Phys. Rep. 121, 263 (1985) . N. Turok and P. Bhattacharjee, Phys. Rev. D 291557 (1984).

8. H. J. de Vega and I. L. Egusquiza, LPTHE preprint 93/43.

9. N. Turok, Phys. Rev. Lett. 60, 549 (1988).

J. D. Barrow, Nucl. Phys. B310, 743 (1988).

\section{FIGURE CAPTIONS}

Fig.1. The time coordinate $q_{0}(\tau)$ for the solution $q_{-}(\sigma, \tau)$ as a function of $\tau$. Since two values of $\tau$ correspond to each value of $q_{0}$, the solution $q_{-}(\sigma, \tau)$ describes two strings. 